\title{
Tuning crystal polymorphs of a $\pi$-extended tetrathiafulvalene-based cruciform molecule towards high-performance organic field-effect transistors
}

\author{
Linlin Feng ${ }^{1,2}$, Huanli Dong ${ }^{2 *}$, Qingyuan $\mathrm{Li}^{1,2}$, Weigang Zhu ${ }^{2}$, Gege Qiu ${ }^{1,2}$, Shang Ding ${ }^{1,2}$, Yang $\mathrm{Li}^{2}$, \\ Mikkel A. Christensen ${ }^{3}$, Christian R. Parker ${ }^{3}$, Zhongming $\mathrm{Wei}^{4}$, Mogens Brøndsted Nielsen ${ }^{3}$ and \\ Wenping $\mathrm{Hu}^{2,5}$
}

\begin{abstract}
It is a common phenomenon for organic semiconductors to crystallize in two or more polymorphs, leading to various molecular packings and different charge transport properties. Therefore, it is a crucial issue of tuning molecular crystal polymorphs (i.e., adjusting the same molecule with different packing arrangements in solid state) towards efficient charge transport and high performance devices. Here, the choice of solvent had a marked effect on controlling the growth of $\alpha$-phase ribbon and $\beta$-phase platelet during crystallization for an indenofluorene (IF) $\pi$-extended tetrathiafulvalene (TTF)-based cruciform molecule, named as IF-TTF. The charge carrier mobility of the $\alpha$-phase IF-TTF crystals was more than one order of magnitude higher than that of $\boldsymbol{\beta}$-phase crystals, suggesting the importance of reasonably tuning molecular packing in solid state for the improvement of charge transport in organic semiconductors.
\end{abstract}

Keywords: organic semiconductor, crystal polymorphs, tetrathiafulvalene-based cruciform molecule, field-effect transistor, charge carrier mobility

Functional organic molecules that may serve as the active components for organic electronics, such as organic field-effect transistors (OFETs), switches, or rectifiers has attracted wide interest in recent years [1-4]. Among them, tetrathiafuvalene (TTF), first discovered by Wudl et al. [5] in 1970, has been seen as a promising building block for developing molecular electrical wires [6] and high performance organic semiconductors because of its unique redox-active properties, being reversibly oxidized in two one-electron steps [7-10] and having a nearly planar molecular structure and strong intermolecular $\pi-\pi$ and S...S interactions facilitating charge transport. To date, a series of TTF-based organic semiconductors with linear-extended conjugation have been synthesized, and excellent charge carrier transport properties in OFETs [1,2,11-15] and molecular devices [16] were demonstrated. Different from the one-dimensional TTF-based molecules widely investigated in the past, we focused on combining dithiafulvene (DTF; "TTF half unit") and other functional molecular units to design cruciform-like molecular structures (that is, two orthogonally oriented $\pi$-systems). One aim was to achieve good and tunable conducting properties by virtue of the different conjugation pathways that exist between the individual parts of the molecule [17-23]. These DTF-based cruciform molecular wires have demonstrated potential promising applications in molecular electronics and also provided a good platform for the investigation of the dependence of conducting properties on their molecular structures [24-26]. As a continuation of our work on such extended TTF compounds, it is interesting to further investigate their applications in organic electronic devices, such as OFETs, the basic component of organic circuits. Here, we present a comprehensive study

\footnotetext{
${ }^{1}$ Beijing Key Laboratory for Optical Materials and Photonic Devices, Department of Chemistry, Capital Normal University, Beijing 100048, China ${ }^{2}$ Beijing National Laboratory for Molecular Sciences, Key Laboratory of Organic Solids, Institute of Chemistry, Chinese Academy of Sciences, Beijing 100190, China

${ }^{3}$ Department of Chemistry, University of Copenhagen, Universitetsparken 5, DK-2100, Copenhagen $\varnothing$, Denmark

${ }^{4}$ State Key Laboratory of Superlattices and Microstructures, Institute of Semiconductors, Chinese Academy of Sciences, Beijing 100083, China

${ }^{5}$ Tianjin Key Laboratory of Molecular Optoelectronic Sciences, Department of Chemistry, School of Sciences, Tianjin University, \& Collaborative Innovation Center of Chemical Science and Engineering, Tianjin 300072, China

*Corresponding author (email: dhl522@iccas.ac.cn)
} 
on an indenfluorene-extended TTF recently reported [23] (IF-TTF; Fig. 1a), tuning its crystal polymorphs, analyzing their molecular structures and measuring their electrical transport properties. The results demonstrated that the obtained charge carrier mobility of the $\alpha$-phase IF-TTF ribbon crystals based-single crystal organic transistors was more than one order of magnitude higher than that of $\beta$-phase platelet crystals, with the highest charge carrier mobility over $1 \mathrm{~cm}^{2} \mathrm{~V}^{-1} \mathrm{~s}^{-1}$, indicating the potential applications of the DTF-based $\pi$-extended cruciform molecules in OFETs and the importance of tuning molecular crystal polymorphs for improvement of their electrical performance.

To probe the effect of DTF units on the electron distribution, the highest occupied molecular orbital (HOMO) and the lowest unoccupied molecular orbital (LUMO) of IF-TTF are shown in Fig. 1b. It can be seen that on HOMO and LUMO orbitals, the electron distribution on the whole molecule demonstrates a quasi-two-dimensional (2D) distribution, suggesting the extended $\pi$-conjugation on the whole molecular structure. It also should be stated that a stronger electron-weight is distributed along the direction of DTF-substituted vertical conjugation backbones, indicating the ability of losing electrons of IF-TTF molecules to the electrodes for the formation of efficient hole charge transport channels in organic electronic devices. The calculated HOMO and LUMO energy levels are $-4.79 \mathrm{eV}$ and $-1.62 \mathrm{eV}$, respectively, with the corresponding experimental values of $-4.64 \mathrm{eV}$ for HOMO and $-2.09 \mathrm{eV}$ for LUMO obtained through the cyclic voltammetry curves [23]. Additionally, a good thermal stability with the decomposition at around $310^{\circ} \mathrm{C}$ for IF-TTF was observed by thermo-gravimetric analysis (TGA) (see Fig. S1, Supplementary Information).

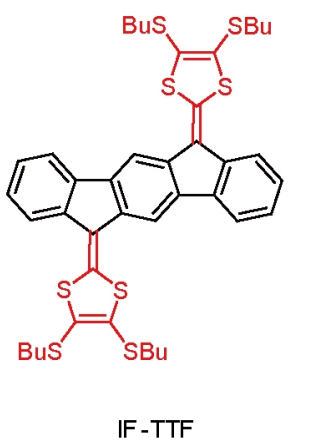

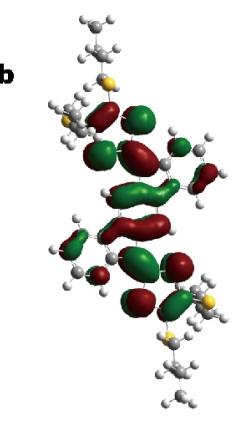

HOMO

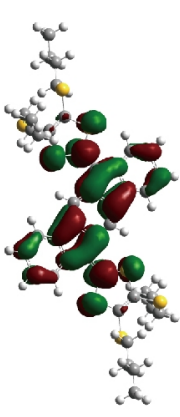

LUMO
Figure 1 (a) Molecular structure of IF-TTF. (b) The calculated HOMO and LUMO orbitals of IF-TTF based on B3LYP/6-31G(d,p) [27].
The extended 2D $\pi$-conjugation of IF-TTF (proposed from the $2 \mathrm{D}$ electron distribution and the good coplanar conjugated structure obtained from its single crystal data as following), the appropriate energy levels, and the good thermal stability of IF-TTF indicate its promising applications in electronic devices. To investigate the intrinsic charge transport of IF-TTF compound, micro/nano- single crystals of IF-TTF were prepared, as we know that organic single crystals are regarded as the best candidates for correctly evaluating the charge transport property of semiconducting materials due to their perfect molecular packing and no grain boundaries, low-density defects [28-30]. Very interestingly, two different crystals phases of IF-TTF could be easily prepared in a good controllability through the simple self-assembly by drop casting IF-TTF solutions, which is independent of the substrates, such as bare $\mathrm{SiO}_{2}$ and octadecyltrichlorosilane (OTS)-modified $\mathrm{SiO}_{2}$ substrates. It was found that when with chloroform as the solvent, the ribbon-shaped $\alpha$-phase IF-TTF single crystals were generally obtained in large area with the ultra-long length of several hundreds of micrometers or even beyond one millimeter and width below several micrometers, corresponding to a high length/width ratio of largely over 100 (Fig. 2a). However, using toluene as the solvent promoted the platelet/block-shaped $\beta$-phase, and IF-TTF single crystals were formed generally with length of tens micrometers and width of several micrometers (Fig. 2b), indicating the strong solvent effect on molecular aggregation [23] and further assembly for IF-TTF, which is very popular for TTF-derived molecules [31-33] and other organic systems [34-36]. Fortunately, by carefully controlling the slow solvent evaporation from their corresponding saturated chloroform or toluene solution for crystal growth, enough large ribbon (named as $\alpha$-phase) and block (named as $\beta$-phase) single crystals of IF-TTF were successfully obtained separately for single crystal data analysis. The X-ray crystallographic results demonstrate that the $\alpha$-phase crystal (obtained based on crystal with size of $0.38 \mathrm{~mm} \times 0.06 \mathrm{~mm} \times 0.03 \mathrm{~mm}$ ) belongs to triclinic $P$-1(2) space group with lattice constants of $a=5.36 \AA$, $b=13.52 \AA, c=14.01 \AA$ and $\alpha=102.98^{\circ}, \beta=94.59^{\circ}, \gamma$ $=90.17^{\circ}$ (CCDC: 1505740$)$. The $\beta$-phase IF-TTF single crystal belongs to triclinic $P-1(2)$ space group, with lattice constants of $a=8.40 \AA, b=9.04 \AA, c=14.00 \AA$ and $\alpha$ $=103.45^{\circ}, \beta=106.21^{\circ}, \gamma=94.23^{\circ}$ (CCDC: 962757) [23]. Good coplanar conjugation between the DTF and IF units in one IF-TTF molecule is demonstrated by the detailed structure analysis, with different angles of $103.28^{\circ}$ and $99.97^{\circ}$ between the molecular conjugated plane and the 
a
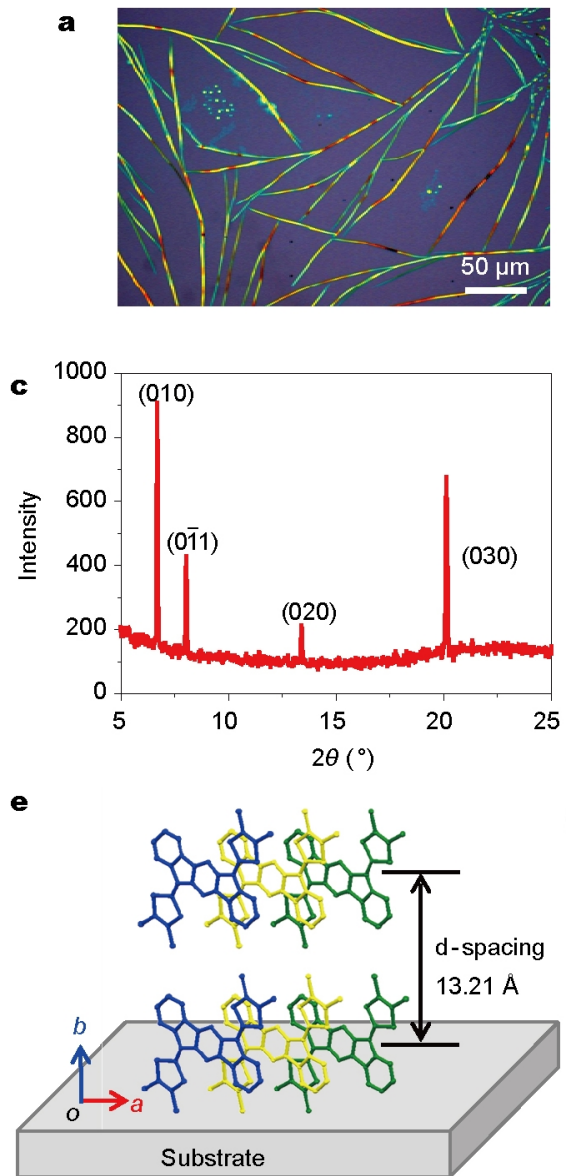

$\mathbf{b}$
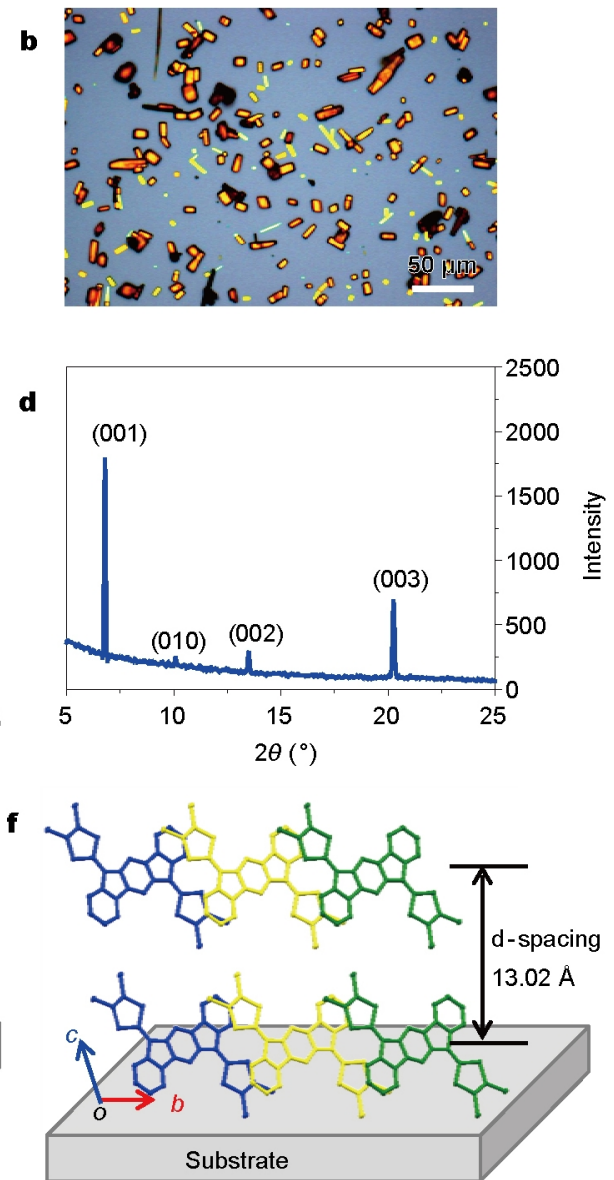

Figure 2 (a, b) Optical microscopy images and (c, d) XRD patterns of the $\alpha$-phase ribbon and $\beta$-phase block IF-TTF crystals. (e, f) The proposed preferable molecular packing modes on the substrate achieved from the XRD results.

$\mathrm{S}$-Bu substituent groups that are out of the planes for $\alpha$-phase and $\beta$-phase crystals, respectively (Fig. S2). Just such a slightly unit cell structure results in obviously different molecular interactions/packing in their crystals and crystal growth behaviors. In $\alpha$-phase crystals, one IF-TTF molecule is connected with six neighboring molecules through strong $\pi \cdots \pi, \mathrm{S} \cdots \mathrm{S}, \mathrm{H} \cdots \mathrm{H}, \mathrm{C}-\mathrm{H} \cdots \pi$ interactions, demonstrating a layer-by-layer molecular packing with discrete columns (Figs S3a-d). The spacing distance of two adjacent columns is $13.52 \AA$ and the interlayer $\pi-\pi$ stacking distance is around $3.375 \AA$. The slipping angle created by the disposition of centroid of the fused ring planes is $44.05^{\circ}$ (Fig. S3d). In comparison, in $\beta$-phase single crystals, one IF-TTF molecule is connected with six neighboring molecules through two types of $\mathrm{C}-\mathrm{H} \cdots \pi$ and $\mathrm{H} \cdot \cdots \mathrm{H}$ interactions (Figs S3e-h). Compared to $\alpha$-phase crystals, there are no S...S interactions observed in $\beta$-phase crystal phase and relatively larger $\pi \cdots \pi$ stacking distance of $3.52 \AA$ coming from the overlap of one end benzene ring and DTF unit between the neighboring molecules. The spacing distance of two adjacent columns and two adjacent layers are $8.05 \AA$ and $13.08 \AA$, respectively. The slipping angle created by the disposition of centroid of the fused ring planes is $67.07^{\circ}$ (Fig. S3h).

The characteristic X-ray diffraction (XRD) patterns of two phases for IF-TTF crystals are shown in Figs $2 \mathrm{c}$ and $\mathrm{d}$, showing a series of sharp reflection with the strongest peak at $2 \theta=6.68^{\circ}$ (corresponding to $d=13.21 \AA$ ) and $2 \theta$ $=6.78^{\circ}$ (corresponding to $d=13.02 \AA$ ), respectively. All the peaks in the XRD patterns could be well indexed according to their single crystal data of $\alpha$-phase and $\beta$-phase, respectively, indicating the pure IF-TTF crystal phases obtained by simple selection of the solvents. The results demonstrate that the $(0 k 0)$ planes in $\alpha$-phase crystals and the (00l) planes in $\beta$-phase crystals are preferably parallel to the substrate. The schematic molecular packing structure related to the substrate are shown in Figs 2e and $f$, where the IF-TTF molecules are preferably standing on the substrate with different title angle of $77.9^{\circ}$ in $\alpha$-phase and $73.73^{\circ}$ in $\beta$-phase, respectively, forming the typical 
layer-by-layer packing structure. To probe the molecular growth model, typical transmission electron microscopy (TEM) was further performed. The TEM images of an individual ribbon and platelet crystal of IF-TTF and their corresponding selected-area electron diffraction (SAED) pattern are shown in Figs $3 a$ and $b$, respectively. During the experiment, no change of the SAED patterns was observed along the whole ribbon and platelet crystals, indicating the single crystal characteristic for both ribbon and platelet crystals. According to the single crystal data of a-phase crystals, its SAED pattern could be well indexed with diffraction spots from the ac planes, agreeing with that $(0 k 0)$ planes parallel to the substrate obtained from its XRD patterns. Detailed analysis indicate that the single crystal ribbons grow along the [100] direction. Additionally, the index of SAED patterns for $\beta$-phase crystals indicates its crystal growth direction along the [010] axis. The [100] direction in $\alpha$-phase and [010] in $\beta$-phase crystals are both corresponding to their $\pi-\pi$ stacking directions, indicating efficient charge transport along the direction (Figs $3 \mathrm{c}$ and $d)$. Additionally, the different $\pi-\pi$ stacking distance and overlaps of $\alpha$-phase and $\beta$-phase crystals along the charge transport direction (Figs $3 \mathrm{c}-\mathrm{f}$ ) suggest their different transporting properties, which provides us a good platform for the investigation of stacking structure and property relationship. Moreover, both the crystal surface
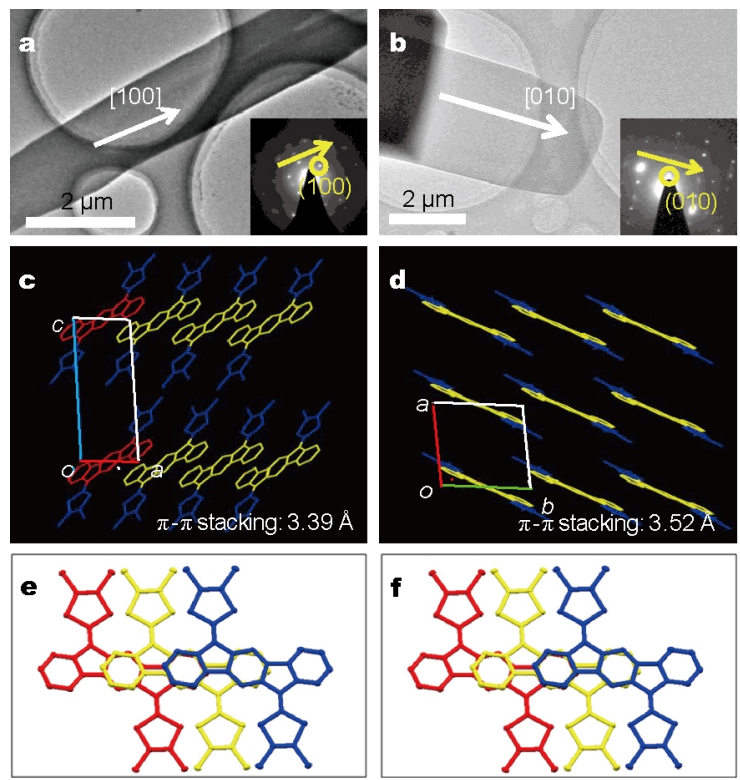

Figure 3 (a, b) TEM images of $\alpha$-phase and $\beta$-phase IF-TTF crystals and their corresponding SAED patterns. (c, d) The deduced molecular packings in $\alpha$ and $\beta$-phase crystals. (e,f) The $\pi$ - $\pi$ stacking overlap in $\alpha$-phase and $\beta$-phase crystals seen along their growth direction axis. of $\alpha$-phase and $\beta$-phase of IF-TTF crystals are very smooth as estimated by atomic force microscopy (AFM), which is beneficial for good contact and electron injection in devices (Fig. S4).

To investigate the charge transport of IF-TTF and elucidate the relationship between molecular packing structures and properties in $\alpha$-phase and $\beta$-phase single crystals, top-contact bottom-gate IF-TTF single crystal transistors were fabricated based on the OTS-modified $\mathrm{Si} / \mathrm{SiO}_{2}$ substrates $\left(\mathrm{SiO}_{2}\right.$ dielectric thickness: $300 \mathrm{~nm}$ with $\mathrm{C}=11$ $\mathrm{nF} \mathrm{cm}^{-2}$ ). Before the device fabrication, the IF-TTF crystals were first heat treated at $80^{\circ} \mathrm{C}$ for $\alpha$-phase and $120^{\circ} \mathrm{C}$ for $\beta$-phase for $2 \mathrm{~h}$ to remove any residual solvent on the crystals and substrate which could act as the electron traps being unfavorable for charge transport. In order to reduce the damage of heat radiation during vacuum electrodes deposition, we adopted a "gluing gold film" technique developed previously in our group [28] to in-situ construct the top source and drain electrodes on IF-TTF crystals. Additionally, for achieving a good contact between crystals and electrodes in the devices, we preferentially selected the relatively thin IF-TTF crystals, that were generally in blue color simply estimated from the optical microscopy due to different refractive index of the different thick crystals related to the substrate [37]. The optical microscopy images of the typical constructed IF-TTF ribbon and platelet crystal transistors are shown in Figs $4 \mathrm{a}$ and b, respectively. Around 40 devices of $\alpha$-IF-TTF and $\beta$-IF-TTF crystals were fabricated and the corresponding bar charts of the frequency distributions are shown in Fig. S6. Representative output and transfer characteristics of these devices are demonstrated in Figs $4 c-f$. It can be seen that both $\alpha-$ and $\beta$-phase IF-TTF single crystal transistors demonstrate typical p-type field-effect transporting property with good gate voltage modulation. Relatively weak contact resistance and hysteresis were observed from the output and transfer curves (Fig. S5), suggesting the good organic-electrode contact and high-quality interface between IF-TTF crystals and the OTS-modified $\mathrm{SiO}_{2}$ substrate. From the saturation regime of the transfer characteristics, the mobility is calculated according to the linear fitting of $\left(-I_{\mathrm{SD}}\right)^{1 / 2}$ versus $V_{\mathrm{G}}$ curve. The obtained average and highest mobilities for $\alpha$-phase IF-TTF crystal transistors are 0.32 and $1.44 \mathrm{~cm}^{2}$ $\mathrm{V}^{-1} \mathrm{~s}^{-1}$, respectively. While for the $\beta$-phase IF-TTF crystal transistors, the average and highest mobilities are only $3.95 \times 10^{-2}$ and $0.28 \mathrm{~cm}^{2} \mathrm{~V}^{-1} \mathrm{~s}^{-1}$, around one order magnitude lower than that of $\alpha$-phase IF-TTF crystal transistors. All the IF-TTF-based transistors exhibit high source-drain on/off current ratio of over $10^{3}$ and low threshold voltage 
a

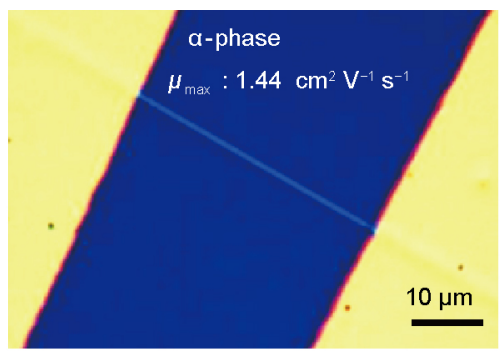

c

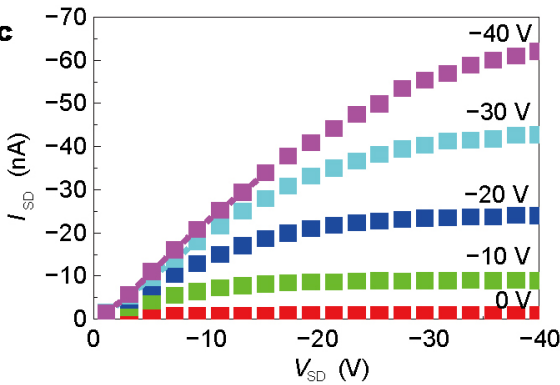

e

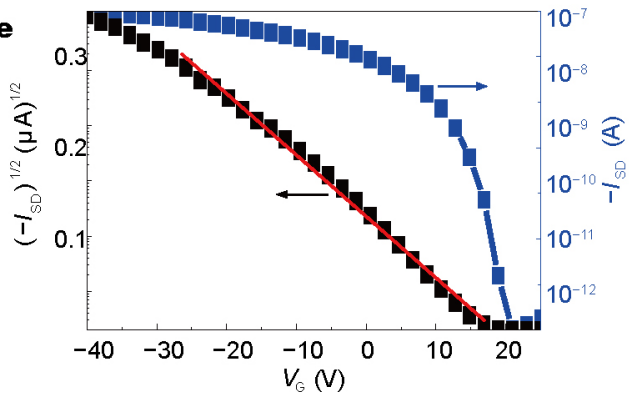

b

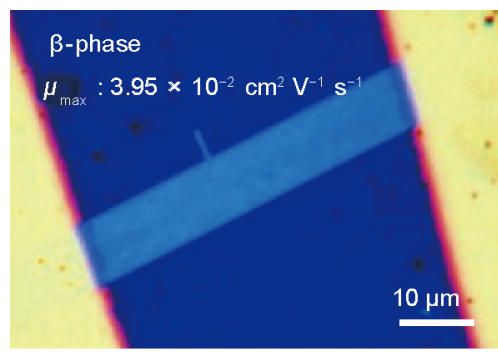

d
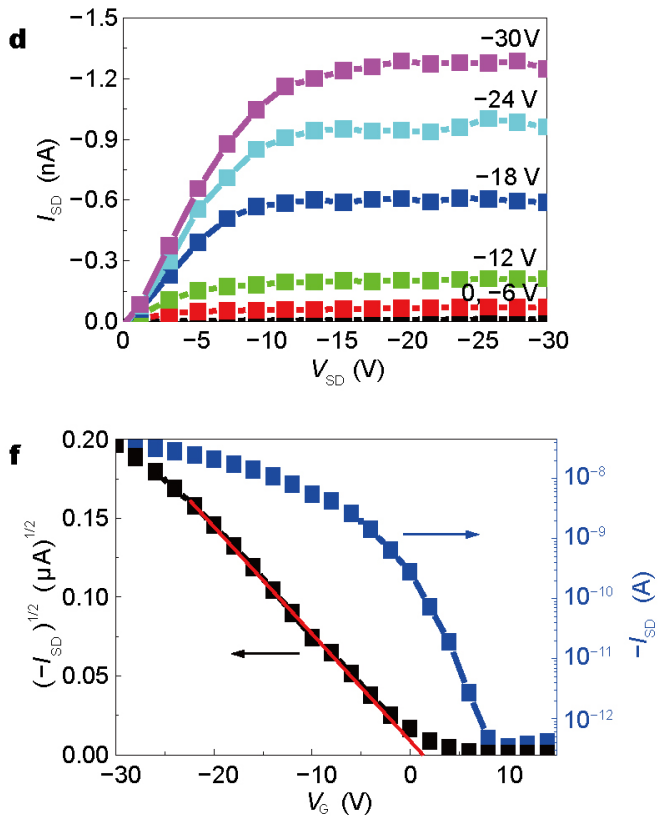

Figure 4 Optical images of device structures based on $\alpha$-phase (a) and $\beta$-phase (b) single crystals. Typical output and transfer characteristics of IF-TTF based transistors based on $(c, e) \alpha$-phase single crystal $\left(V_{\mathrm{SD}}=-40 \mathrm{~V}\right)$ and $(\mathrm{d}, \mathrm{f}) \beta$-phase single crystal $\left(V_{\mathrm{SD}}=-30 \mathrm{~V}\right)$.
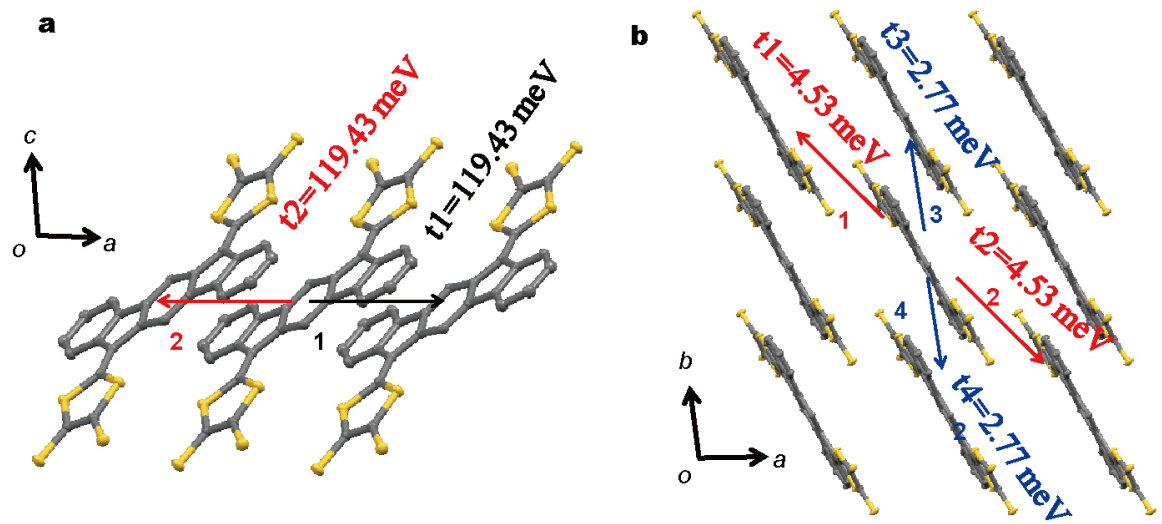

Figure 5 Illustrations of molecular packing structures and transfer integrals for the nearest neighboring molecular pairs considered in the calculations (a) for a-phase crystal and (b) for $\beta$-phase crystal based on the direct integration method $\left(V=\left\langle\Phi_{\text {Номо }}\left|F_{0}\right| \Phi_{\text {номо }}\right\rangle\right)$ with site-energy correction $[38]$.

below $-5 \mathrm{~V}$. The higher charge carrier transport obtained based on $\alpha$-phase IF-TTF crystal transistor is attributed to the more condensed molecular packing in this state, which is consistent with its relatively larger transfer integral $(119.43 \mathrm{meV}$ ) than that of $\beta$-phase crystals (4.53 and 2.77 $\mathrm{meV}$ ) by the theoretical calculations (Fig. 5). More detai- 
Table 1 The comparison of the charge transport property for $\alpha$-phase and $\beta$-phase IF-TTF crystals

\begin{tabular}{|c|c|c|}
\hline Identification code & CCDC: 1505740 (a-phase) & CCDC: 962757 ( $\beta$-phase) \\
\hline Reorganization energy & \multicolumn{2}{|c|}{$326.20 \mathrm{meV}$} \\
\hline Transfer integral (meV) & $\begin{array}{l}\text { Path 1: } 119.43(a) \\
\text { Path 2: } 119.43(-a)\end{array}$ & $\begin{array}{l}\text { Path 1: } 4.53(a-b) \text {, Path 3: } 2.77(b) \\
\text { Path 2: } 4.53(-a+b) \text {, Path 4: } 2.77(-b)\end{array}$ \\
\hline Calculated mobility $\mu\left(\mathrm{cm}^{2} \mathrm{~V}^{-1} \mathrm{~s}^{-1}\right)$ & 0.98 (along $a$ axis) & 0.0037 (along $b$ axis) \\
\hline Measured mobility $\mu_{\max }\left(\mathrm{cm}^{2} \mathrm{~V}^{-1} \mathrm{~s}^{-1}\right)$ & 1.44 (along $a$ axis) & 0.28 (along $b$ axis) \\
\hline
\end{tabular}

led comparison information for $\alpha$-phase and $\beta$-phase crystals of IF-TTF are summarized in Table 1. Here for the first time the achieved high charge carrier mobility, on/off ratio and the low threshold voltage of DTF-derivative-based transistors suggest the promising potential applications of DTF-extended organic semiconductors in OFETs with the consideration of rationally controlling their crystal polymorphs.

In summary, two crystal polymorphs of IF-TTF were controllably achieved through the simple solvent selection, the structures of which were comprehensively characterized. The crystal polymorph-dependent carrier mobility was investigated based on their single crystals field-effect transistors. Compared with $\beta$-phase IF-TTF single crystals, the $\alpha$-phase IF-TTF single crystals demonstrate more than one order of magnitude higher charge carrier mobility with the highest carrier mobility value around $1.44 \mathrm{~cm}^{2} \mathrm{~V}^{-1} \mathrm{~s}^{-1}$ and the on/off ratio of $10^{3}$, suggesting the potential application of such type semiconductors in OFETs. This study also provides the guideline for tuning molecular crystal polymorph and molecular structure design for achieving high electrical property.

Received 10 October 2016; accepted 31 October 2016; published online 6 December 2016

1 Dong $\mathrm{H}$, Wang $\mathrm{C}, \mathrm{Hu}$ W. High performance organic semiconductors for field-effect transistors. Chem Commun, 2010, 46: 5211-5222

2 Wang $\mathrm{C}$, Dong $\mathrm{H}, \mathrm{Hu} \mathrm{W}$, et al. Semiconducting $\pi$-conjugated systems in field-effect transistors: a material odyssey of organic electronics. Chem Rev, 2012, 112: 2208-2267

3 Qian Y, Zhang X, Qi D, et al. Thin-film organic semiconductor devices: from flexibility to ultraflexibility. Sci China Mater, 2016, 59: $589-608$

4 Qin X, Dong H, Hu W. Green light-emitting diode from bromine based organic-inorganic halide perovskite. Sci China Mater, 2015, 58: $186-191$

5 Wudl F, Smith GM, Hufnagel EJ. Bis-1,3-dithiolium chloride: an unusually stable organic radical cation. J Chem Soc D, 1970, 1453-1454

6 Wei Z, Hansen T, Santella M, et al. Molecular heterojunctions of oligo(phenylene ethynylene)s with linear to cruciform framework. Adv Funct Mater, 2015, 25: 1700-1708

7 Segura JL, Martín N. New concepts in tetrathiafulvalene chemistry.
Angew Chem Int Ed, 2001, 40: 1372-1409

8 Ho G, Heath JR, Kondratenko M, et al. The first studies of a tetrathiafulvalene- $\sigma$-acceptor molecular rectifier. Chem Eur J, 2005, 11: 2914-2922

9 Mas-Torrent M, Rovira C. Novel small molecules for organic fieldeffect transistors: towards processability and high performance. Chem Soc Rev, 2008, 37: 827-838

10 Bendikov M, Wudl F, Perepichka DF. Tetrathiafulvalenes, oligoacenenes, and their buckminsterfullerene derivatives: the brick and mortar of organic electronics. Chem Rev, 2004, 104: 4891-4946

11 Tucker NM, Briseno AL, Acton O, et al. Solvent-dispersed benzothiadiazole-tetrathiafulvalene single-crystal nanowires and their application in field-effect transistors. ACS Appl Mater Interfaces, 2013, 5: 2320-2324

12 Jiang H, Yang X, Wang E, et al. Organic single crystalline microand nanowires field-effect transistors of a tetrathiafulvalene (TTF) derivative with strong $\pi-\pi$ orbits and $S \cdots$ S interactions. Synth Met, 2011, 161: 136-142

13 Leufgen M, Rost O, Gould C, et al. High-mobility tetrathiafulvalene organic field-effect transistors from solution processing. Org Electron, 2008, 9: 1101-1106

14 Yang G, Di C, Zhang G, et al. Highly sensitive chemical-vapor sensor based on thin-film organic field-effect transistors with benzothiadiazole-fused-tetrathiafulvalene. Adv Funct Mater, 2013, 23: 1671-1676

15 Mas-Torrent M, Masirek S, Hadley P, et al. Organic field-effect transistors (OFETs) of highly oriented films of dithiophene-tetrathiafulvalene prepared by zone casting. Org Electron, 2008, 9: 143-148

16 Wang Z, Dong H, Li T, et al. Role of redox centre in charge transport investigated by novel self-assembled conjugated polymer molecular junctions. Nat Commun, 2015, 6: 7478

17 Jennum K, Nielsen MB. Tetrathiafulvalene-based cruciform molecules. Chem Lett, 2011, 40: 662-667

18 Nielsen MB. Molecular scaffolding with tetrathiafulvalene-design and synthesis of new molecules for molecular electronics. Phosphorus Sulfur Silicon Related Elements, 2011, 186: 1055-1073

19 Sørensen JK, Vestergaard M, Kadziola A, et al. Synthesis of oligo(phenyleneethynylene)-tetrathiafulvalene cruciforms for molecular electronics. Org Lett, 2006, 8: 1173-1176

20 Vestergaard M, Jennum K, Sørensen JK, et al. Synthesis and characterization of cruciform-conjugated molecules based on tetrathiafulvalene. J Org Chem, 2008, 73: 3175-3183

21 Lincke K, Floor Frellsen A, Parker CR, et al. A tetrathiafulvalenefunctionalized radiaannulene with multiple redox states. Angew Chem Int Ed, 2012, 51: 6099-6102

22 Christensen MA, Rudebusch GE, Parker CR, et al. Diindenothienoacene-tetrathiafulvalene redox systems. RSC Adv, 2015, 5: 49748-49751 
23 Christensen MA, Parker CR, Sørensen TJ, et al. Mixed valence radical cations and intermolecular complexes derived from indenofluorene-extended tetrathiafulvalenes. J Mater Chem C, 2014, 2 : 10428-10438

24 Parker CR, Wei Z, Arroyo CR, et al. A new class of extended tetrathiafulvalene cruciform molecules for molecular electronics with dithiafulvene-4,5-dithiolate anchoring groups. Adv Mater, 2013, 25: 405-409

25 Parker CR, Leary E, Frisenda R, et al. A comprehensive study of extended tetrathiafulvalene cruciform molecules for molecular electronics: synthesis and electrical transport measurements. J Am Chem Soc, 2014, 136: 16497-16507

26 Lissau H, Frisenda R, Olsen ST, et al. Tracking molecular resonance forms of donor-acceptor push-pull molecules by singlemolecule conductance experiments. Nat Commun, 2015, 6: 10233

27 Gaussian 09, Frisch MJ, Trucks GW, Schlegel HB, Gaussian, Inc., Wallingford CT, 2009

28 Tang Q, Jiang L, Tong Y, et al. Micrometer- and nanometer-sized organic single-crystalline transistors. Adv Mater, 2008, 20 : 2947-2951

29 Jiang H, Kloc C. Single-crystal growth of organic semiconductors. MRS Bull, 2013, 38: 28-33

30 Yao Y, Dong $\mathrm{H}, \mathrm{Hu} \mathrm{W}$. Charge transport in organic and polymeric semiconductors for flexible and stretchable devices. Adv Mater, 2016, 28: 4513-4523

31 Jiang H, Yang X, Cui Z, et al. Phase dependence of single crystalline transistors of tetrathiafulvalene. Appl Phys Lett, 2007, 91: 123505

32 Pfattner R, Mas-Torrent M, Bilotti I, et al. High-performance single crystal organic field-effect transistors based on two dithiophenetetrathiafulvalene (DT-TTF) polymorphs. Adv Mater, 2010, 22: 4198-4203

33 Brillante A, Bilotti I, Della Valle RG, et al. The four polymorphic modifications of the semiconductor dibenzo-tetrathiafulvalene. CrystEngComm, 2008, 10: 1899-1909
34 He P, Tu Z, Zhao G, et al. Tuning the crystal polymorphs of alkyl thienoacene via solution self-assembly toward air-stable and highperformance organic field-effect transistors. Adv Mater, 2015, 27: 825-830

35 Wang C, Liu Y, Wei Z, et al. Biphase micro/nanometer sized single crystals of organic semiconductors: control synthesis and their strong phase dependent optoelectronic properties. Appl Phys Lett, 2010, 96: 143302

36 Law KY. Organic photoconductive materials: recent trends and developments. Chem Rev, 1993, 93: 449-486

37 Jiang L, Hu W, Wei Z, et al. High-performance organic single-crystal transistors and digital inverters of an anthracene derivative. Adv Mater, 2009, 21: 3649-3653

38 Shuai Z, Wang D, Peng Q, et al. Computational evaluation of optoelectronic properties for organic/carbon materials. Acc Chem Res, 2014, 47: 3301-3309

Acknowledgments The work was supported by Beijing NOVA Programme (Z131101000413038), Beijing Local College Innovation Team Improve Plan (IDHT20140512), the National Natural Science Foundation of China $(91433115,91222203,91233205$ and 51222306), the Ministry of Science and Technology of China (2013CB933403 and 2013CB933504) and the University of Copenhagen.

Author contributions Dong $\mathrm{H}$ and $\mathrm{Hu} \mathrm{W}$ conceived and designed the experiments. Christensen MA, Parker CR and Nielsen MB synthesized the compound. Feng L performed the experiments. Zhu W contributed to the structure analysis. Li Y contributed to the theoretical calculations. Dong $\mathrm{H}$, Feng L, Hu W and Nielsen MB co-wrote and revised the manuscript. All the authors contributed to the general discussion.

Conflict of interest The authors declare that they have no conflict of interest.

Supplementary information The experiment data are available in the online version of the paper. 

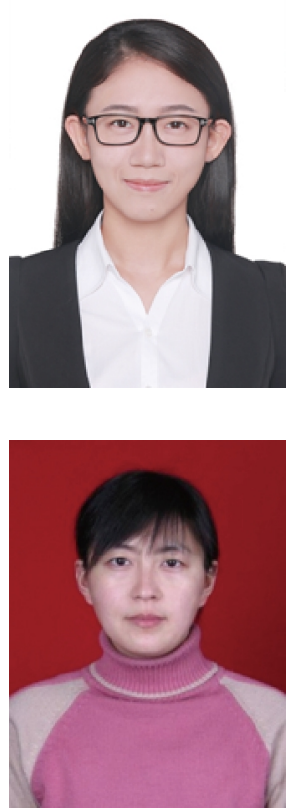

Linlin Feng obtained her BSc degree from Capital Normal University (CNU) in 2014 and now is a graduate student at the Department of Chemistry, CNU and majored in polymer chemistry and physics. Her research interest is in conjugated polymer materials and devices.
Huanli Dong received her PhD degree (2009) of physical chemistry from the Institute of Chemistry, Chinese Academy of Sciences (ICCAS). She is now a professor of the Key Laboratory of Organic Solids, ICCAS. She received the Outstanding Young Scientist Foundation of NSFC (2012), the Prize for Young Chemists of Chinese Chemical Society (2014). Her research interests include molecular materials, crystals and devices of organic/polymeric semiconductors, and currently she has published more than 110 peer-reviewed papers with citation over 3000 times.

\section{通过晶相调控实现高性能十字形TTF分子场效应晶体管器件构筑}

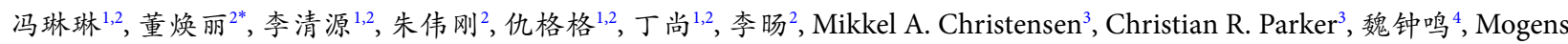
Brøndsted Nielsen ${ }^{3}$, 胡文平 2,5

摘要 有机分子溶剂的选择对于调控不同晶相来说有着关键性作用. 本文通过两种溶剂的调控, 成功得到荫并芴四硫富瓦烯(IF-TTF)两种 不同的晶相一 $\alpha$ 相带状晶体和 $\beta$ 相片状晶体, 并对两种晶相的内部分子排列堆积情况进行了一系列的对比分析. 结果表明场效应电荷传输 能力随着晶相的不同而有所差异, 直接证明了分子堆积的合理调控对实现有机半导体材料高性能电荷传输性能的重要性. 